ESTUDIO DE CASOS Rev Chil Salud Pública 2013 Vol 17 (2): 139-146

Consuelo Cárdenas ${ }^{1}$ Carolina Pinninghoff ${ }^{2}$

'Programa de Magister y Especialización en Terapia Familiar Sistémica. Universidad de La Frontera, Departamento de Psicología. Temuco. consuelo.cardenas.ps@gmail.com ${ }^{2}$ Programa de Magister y Especialización en Terapia Familiar Sistémica. Universidad de La Frontera, Departamento de Psicología. Temuco. ps.pinninghoff@gmail.com

\section{Modelo integral de salud con Enfoque Familiar y Comunitario: Experiencias en la implementación desde un equipo de atención hospitalaria}

\section{RESUMEN}

Esta investigación describe las experiencias de un equipo de atención hospitalaria en torno a la implementación del Modelo Integral de Salud Familiar y Comunitario. Estudio exploratorio de carácter cualitativo. Se realizaron 2 entrevistas grupales semi-estructuradas a 17 miembros del Equipo del Hospital Comunitario de Salud Familiar de El Carmen, provincia de Nuble, los cuales se dividieron en dos grupos de 8 y 9 personas respectivamente. Los resultados señalan la relevancia de considerar en la Implementación del Modelo, Capacidad de organización del equipo, Autonomía en la toma de decisiones, Confianza del equipo en sus capacidades individuales y colectivas, Comprensión del Modelo de Salud Familiar y Convicción en la viabilidad de éste.

Palabras Clave: Modelo de Salud Familiar, Equipo de Salud Hospitalario, Etapas en la Implementación del Modelo de Salud Familiar.

\section{COMPREHENSIVE HEALTHCARE WITH A FAMILY AND COMMUNITY FOCUS: EXPERIENCES FROM A HOSPITAL-BASED HEALTHCARE TEAM}

\section{ABSTRACT}

This investigation describes the experiences of a hospital-based healthcare team, with the implementation of the Comprehensive Family and Community Healthcare Model. This was an exploratory study of qualitative nature. Two semi-structured group interviews were carried out with a team of 17 workers at the El Carmen Family Health Community Hospital, in the province of $\tilde{N}$ uble, divided in two groups of 8 and 9 persons respectively. The results highlight the importance of considering, when implementing such a program, factors to such as the team's organizational capacities, autonomy in the decision-making process, the team's confidence in their own individual and collective capacities, comprehension of the Family Healthcare Model and belief in the model's viability.

Key words: Family Health Model, Hospital-based Healthcare Team, Family Healthcare Model Steps in Implementation. 


\section{INTRODUCCIÓN}

La implementación de modelos basados en Medicina y Salud Familiar ha sido una de las temáticas recurrentes en las reformas sanitarias recientes en Latinoamérica ${ }^{1}$.

Chile desde la década de los '90 ha puesto en marcha un proceso de reforma de su sistema de salud, teniendo en consideración los cambios demográficos y epidemiológicos ${ }^{2}$, como también, educacionales, familiares, comunitarios y sociales ${ }^{3}$.

Es así como en el marco de la Reforma Sanitaria del año 2004, el Ministerio de Salud chileno señala: potenciar la estrategia de Atención Primaria en Salud (APS) y cambiar el Modelo de Atención. Surge de esta forma el Modelo de Salud Familiar (MSF), el cual sustentado en los enfoques biopsicosocial y sistémico, pone el acento en las personas y su contexto familiar y social ${ }^{4}$.

En febrero del año 2004, se publica en Chile la Ley de Autoridad Sanitaria N¹9937, la cual da a conocer aspectos del funcionamiento de la red de salud nacional. Dentro de sus especificaciones señala la presencia de Establecimientos de Menor Complejidad: Centros de Atención Primaria y Hospitales de Baja Complejidad, también conocidos como Hospitales de la Familia y la Comunidad (HFC) ${ }^{5}$.

Se ha definido que un (HFC), es un establecimiento que forma parte de la estrategia de atención primaria, con énfasis en la promoción y prevención en salud. Son el punto de contacto más cercano y permanente de la población con su sistema sanitario. Estos establecimientos participan activamente con la comunidad, adaptando sus servicios a los requerimientos de ésta ${ }^{6}$.

A partir de la recopilación efectuada sobre las primeras experiencias de los equipos de salud en APS en torno al desarrollo del MSF chileno, surge en el año 2008 la publicación "En el camino a Centro de Salud Familiar", donde sus autores a modo de esquematizar este proceso señalan la presencia de 5 etapas, siendo éstas: Etapa de Consultorio, Etapa de Motivación y Compromiso, Etapa de Desarrollo, Etapa de Fortalecimiento y Etapa de Consolidación ${ }^{2}$. De las experiencias derivadas de estos equipos de salud, se plantean importante considerar: grado de compromiso y motivación del equipo, madurez y organización de éste, claridad en la comprensión del MSF y la presencia de apoyos externos, entre otros elementos, lo cuales se constituyen como el pilar fundamental en la construcción del MSF chileno?.

Considerando los antecedentes antes expuestos y teniendo presente que la actual reforma de salud chilena apunta a masificar el MSF a toda la red sanitaria, es que el interés de las investigadoras se centra es describir cómo ha sido la implementación del MSF en un equipo de salud el cual se desempeña en el ámbito hospitalario. Es así como el presente estudio intenta responder la siguiente pregunta: ¿Cuáles son las experiencias en la implementación del Modelo Integral con Enfoque Familiar y Comunitario en el Equipo de Salud del Hospital Comunitario de Salud Familiar de El Carmen? siendo sus objetivos:

- Describir cómo el equipo de salud ha implementado el Modelo Integral con Enfoque Familiar Comunitario.

- Describir la vivencia del Equipo de Salud en la implementación del Modelo Integral con Enfoque Familiar y Comunitario.

\section{MATERIAL Y MÉTODO}

Se trata de un estudio exploratorio, de carácter cualitativo. Se realizaron 2 entrevistas grupales semiestructuradas, a 17 miembros del equipo de salud del Hospital Comunitario de Salud Familiar de El Carmen, los cuales se dividieron en dos grupos de 8 y 9 personas respectivamente. Los grupos estuvieron conformados por profesionales, técnicos y administrativos del Hospital. Para la selección de los participantes, se utilizó un muestreo por criterio. En cuanto a los criterios de inclusión fueron considerados: integrantes del equipo de salud del Hospital Comunitario y de Salud Familiar de El Carmen, con formación en el MSF y un año de experiencia en la ejecución de éste.

Siendo la técnica de recolección de la información la entrevista grupal semiestructurada, se elaboró una pauta de entrevista grupal semiestructurada, diseñada por las investigadoras en base a la revisión teórica efectuada y los objetivos propuestos en la investigación. Una vez elaborada se solicitó a grupo experto su evaluación, considerando sus sugerencias se elaboró la versión final de la pauta de entrevista, la cual abordó dos temáticas centrales: 
- Aspectos procedimentales y organizacionales del equipo de salud, en el contexto de la implementación del MSF.

- Vivencias del equipo de salud en torno a la implementación del MSF.

Obtenida la autorización formal por parte del Director del Hospital Comunitario de Salud Familiar de El Carmen, para la realización del estudio, se acuerda que las entrevistas grupales se realizarían a fines del mes de agosto de 2011, previo a la fecha de ejecución de éstas, se envió a encargada del Programa de Salud Comunitaria y Familiar del Hospital de El Carmen, los consentimientos informados, para su distribución a los participantes de la investigación.

Dentro de las consideraciones éticas, se informó a cada uno de los participantes el propósito del estudio, respetando la libertad de participación. Se preservó su privacidad, manteniendo la confidencialidad en el uso de los datos.

Los datos obtenidos se registraron mediante formato de audio-video. Una vez transcritos, fueron codificados, realizándose una triangulación de la información por las investigadoras. Se generó posteriormente una red de categorías las cuales se ordenaron en base a 5 ejes temáticos, cuyo análisis de contenido dio origen a los resultados del estudio.

La devolución de los resultados se realizará al Director del Hospital, como también a los miem- bros del equipo de salud participantes de la investigación, en fecha definida en forma conjunta.

\section{RESULTADOS}

A modo de sistematizar la experiencia vivida por el equipo de salud del Hospital de El Carmen, los resultados se exponen en base al desarrollo de tres fases en la implementación del MSF, siendo éstas: Fase Inicial: El equipo de salud se aproxima a la implementación del MSF en su recinto hospitalario.

Fase Intermedia: El equipo de salud muestra una apropiación del MSF en su Hospital.

Fase Actual: El equipo de salud consolida el MSF en su centro de atención sanitaria.

En cada de las fases antes descritas, es posible reconocer cinco dimensiones: Desarrollo en la Implementación del MSF, Características del Equipo, Vivencia del Equipo, Contexto y Aprendizajes del trabajo con Familias (Figura 1).

\section{Fase Inicial: Aproximación}

\section{Desarrollo en la Implementación del Mo-} delo de Salud Familiar.

El equipo se encuentra en una aproximación al MSF, la cual se caracterizó por una falta de orientación técnica en el MSF y una falta de retroalimentación al equipo desde el Servicio de Salud

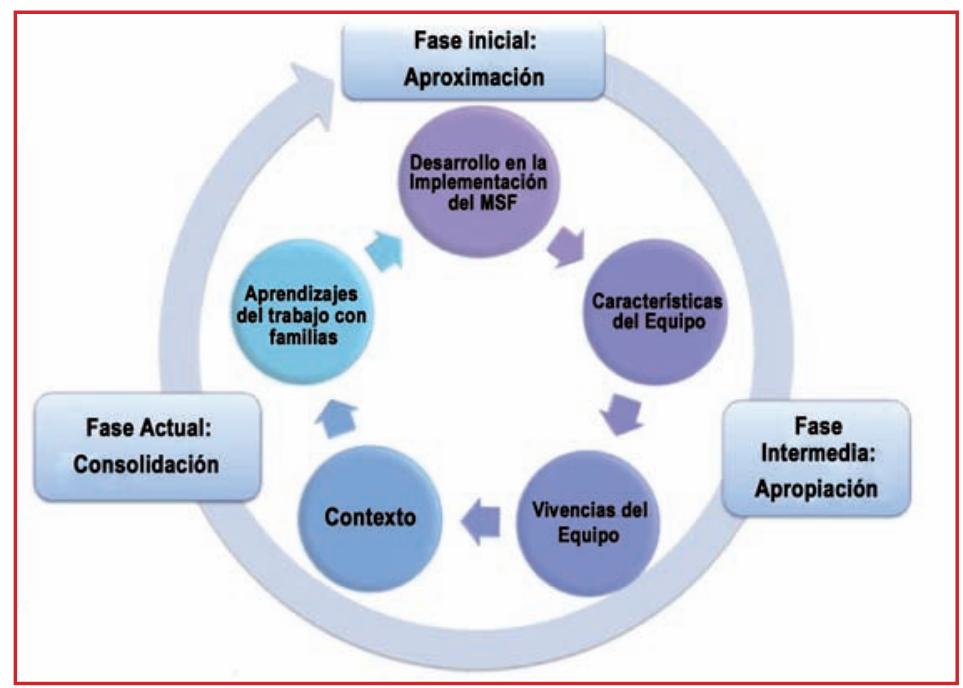

Figura 1. Fases en la Implementación del Modelo de Salud Familiar.

Fuente: Elaboración propia. 
provincial. Algunos miembros del equipo contaban con un conocimiento previo del MSF, derivado de su formación universitaria ligada a carreras del área de las ciencias sociales.

Dada la falta de claridad manifestada en esta etapa del proceso, surge la necesidad desde el propio equipo de generar las primeras directrices del MSF, lo que posteriormente facilitó el surgimiento de las primeras instancias de acuerdo entre éste y el Servicio de Salud provincial.

\section{Caracteristicas del equipo}

El equipo se enfoca a la nueva tarea de llevar a cabo la implementación del MSF, destacándose su gran responsabilidad y persistencia en llevar a cabo este proceso.

La relación entre sus miembros es cercana, respetuosa y afectiva. Se evidencian espacios de encuentro formales e informales, los que actúan como facilitadores de su organización y toma de decisiones, "la mayoría de las personas que trabaja acá tiene lazos emocionales con todos...estamos todos afectuosamente unidos".

Cuentan con un líder motivador, comprometido y asertivo, con conocimiento de las características y habilidades de su equipo. Los miembros del equipo lo consideran como un integrante más, "como uno más del equipo".

\section{Vivencia.}

Desde el equipo se evidencia una sensación de temor asociada al posible cierre del Hospital y cambio de éste a CESFAM y a su vez, por la forma de ejecución del MSF "Cómo lo vamos a hacer".

Surge también una sensación de rechazo en torno a cambios en la modalidad de atención (de individual a familiar).

Por último, se hace manifiesta una sensación de imposición frente a la implementación del MSF, en parte del equipo de salud, a partir de los lineamientos entregados por el Servicio de Salud provincial.

\section{Contexto.}

En esta primera etapa, el equipo señala la ausencia de experiencias nacionales en la implementación del MSF en el ámbito hospitalario, manifestando que sólo contaban con información relativa a experiencias en la implementación del MSF en CESFAM chilenos.

\section{Aprendizajes del trabajo con familias.}

Puesto que la implementación del MSF se encontraba en vías de desarrollo, el equipo aún no visualizaba aprendizajes en el trabajo con familias desde esta modalidad de atención.

\section{Fase Intermedia: Apropiación}

\section{Desarrollo en la Implementación del Modelo de Salud Familiar.}

Se caracteriza por la apropiación del MSF por parte del equipo. Se concretan coordinaciones y acciones, tales como: reuniones mensuales, capacitaciones en torno al MSF y pasantías del MSF, desde el Servicio de Salud Provincial. Se producen instancias de encuentro con otros equipos sanitarios de la región.

$\mathrm{Al}$ interior del Hospital, se establecen cambios administrativos, conformación de subsectores y equipos de trabajo (Equipo Gestor, Equipo Transversal, Equipo sector Azul, Equipo sector Rojo), se diseñan nuevos sistemas de registro familiar tanto escritos como digitales y también, se incorporan nuevos profesionales al establecimiento.

\section{Características del equipo.}

En el diseño de esta nueva forma de hacer salud, se manifiesta la creatividad surgida desde el propio equipo en su forma de organización, difusión e implementación del MSF.

Se manifiesta un alto grado de compromiso en gran parte del equipo a la hora de llevar a cabo la implementación del MSF. Surgen acciones como el "voluntariado", donde miembros del equipo asisten al Hospital, fuera de su horario laboral, para trabajar en el diseño y difusión del MSF, tanto al interior del Hospital como al exterior de éste, en acciones con la comunidad.

El equipo refiere poseer gran confianza en sus capacidades individuales y colectivas para llevar a cabo el desafío de implementar el MSF en su Hospital.

\section{Vivencia}

El equipo manifiesta una sensación de convicción y motivación que la implementación del MSF y su viabilidad en el tiempo son posibles, "hay que hacerlo y hay que hacerlo porque es lo que se viene y no nos podemos quedar atrás, tenemos que ser los mejores y tenemos que ser los primeros y tenemos 
que actuar, porque nosotros somos buenos... un desafío bonito, un desafío a vencernos, a no dejarnos morir, ni aplastar".

\section{Contexto}

En base a los lineamientos generados desde el equipo gestor, comienza a difundirse el MSF a nivel interno a partir de capacitaciones a otros funcionarios. A nivel externo se difunde el MSF a la comunidad a través de visitas domiciliarias y avisos mediante medios de difusión masiva y coordinación con redes locales.

\section{Aprendizaje del trabajo con familias.}

El equipo comienza a visualizar cambios en su forma de relacionarse con las personas que atiende, aproximándose hacia una mirada más integral de los procesos de salud y enfermedad de las personas "este es el modelo necesario para trabajar en salud, que las personas están dentro de un contexto social, que influye en como ellos viven su enfermedad o como se mejoran, más que nada eso, estar conscientes que trabajamos con personas".

\section{Fase Actual: Consolidación}

\section{Desarrollo en la Implementación del Mo- delo de Salud Familiar.}

Se caracteriza por la consolidación del MSF en este equipo de salud. Se manifiesta una sistematización del trabajo ejecutado, lo que se evidencia en la configuración del Hospital como Centro de Pasantía a nivel nacional del MSF, aportando a otros equipos de salud en el conocimiento teórico y experiencial logrado a partir del trabajo con familias.

\section{Caracteristicas del equipo.}

Manifiestan un fortalecimiento de sus habilidades sociales y un mayor grado de cercanía en el contacto y aproximación al trabajo con familias.

Existe una apertura en la comprensión de los procesos de salud y enfermedad de quienes atienden desde una mirada más integral: "porque las personas no sólo somos biológicas sino que estamos insertas en un sistema familiar que si o si afecta nuestra situación de salud-enfermedad".

Señalan poseer un mayor dominio y utilización de elementos del enfoque sistémico aplicando conceptos tales como: tipos de familia, dinámica familiar, estructura familiar, vínculos, roles familiares, etc., los cuales utilizan a la hora de trabajar multidisciplinariamente, tanto en las reuniones de subsectores, como en el trabajo directo con familias.

Surge el desgaste por estrés laboral, el cual comienza a generar ausentismo laboral y rotación de profesionales. El equipo se organiza y comienza a generar sus propias estrategias de autocuidado.

Respecto a los problemas derivados del trabajo en equipo, mencionan la impuntualidad en los espacios de encuentro señalando también: "nuestra dificultad parece ser que nos cuesta encontrar dificultades".

\section{Vivencia.}

Gran parte del equipo señala sentir lealtad hacia el hospital y las familias que atienden "este hospital ha sido como la vida misma, un terreno donde aprender y donde tener una misión también dentro de eso".

A partir de la intervención con familias, mencionan sentir satisfacción personal y profesional, dada la relación establecida con éstas, "ha sido muy enriquecedor, porque llegar a una familia, que te abra la puerta de su casa para poder intervenir ahí, además la gente se siente súper parte, nosotros también muy parte de su familia".

\section{Contexto}

Se señala al espacio territorial como favorecedor de los procesos de salud. Muchos de los integrantes del equipo de salud son miembros de la propia comunidad, estableciéndose sistemas relacionales en otros espacios de encuentro, los cuales han facilitado el despliegue de iniciativas de promoción y prevención en salud, en donde el equipo hospitalario enfatiza la participación de las personas en su propio cuidado sanitario. "Uno va saliendo y se encuentra con alguien que te ubica. Hay mucha gente que es de acá reside en el Carmen o ha vivido muchos años y conoce a la gente y a las historias familiares lo que con el modelo o sin el modelo hay cosas que se dan".

Otro elemento importante ha sido el ordenamiento territorial a través de subsectores, lo cual ha permitido que la propia comunidad vaya desarrollando un sentido de pertenencia, a partir de una vinculación física y relacional con el subsector 
al cual se encuentra adscrita. "...ha sido bonito que se identifiquen con nosotros, la señorita dice me atiendo como usted no más".

\section{Aprendizaje del trabajo con familias.}

Desde el equipo se ha visto una amplitud del concepto de familia, definiéndolo como: "red de vínculos afectivos entre sus miembros, más que la relación de consanguineidad que pueda existir entre éstos".

En lo que respecta al aprendizaje desde la intervención directa con familias, manifiestan: "en la intervención con familias hay ausencia de absolutos".

Comprenden su intervención como un apoyo más que como una imposición, "el modelo va enfocado a otra cosa, no va a mantener este tema asistencialista, sino va a lograr que las personas logren empoderarse de su situación".

Manifiestan un giro en su modalidad de atención desde: "atender al paciente a atender a la persona".

Finalmente, el equipo de personas que conforman el Hospital de El Carmen, refieren estar avanzando en la comprensión e integralidad de los procesos de salud desde una manera más amable y cercana a las personas, viéndose reflejados ellos mismos en muchos de los procesos que a diario viven junto a las familias que atienden.

“...si una familia viene a nosotros, lo primero que necesita es ser acogida, comprendida, es decir, si ellas van al equipo tanto es porque tienen un problema y esperan ser acogidos de buena forma, escuchados de buena forma, que los orienten, y que al momento de salir del establecimiento de salud puedan decir que conversaron con un equipo que los atendió".

\section{DISCUSIÓN}

Algunos elementos que hicieron posible la implementación del MSF en Hospital del Carmen fueron: Capacidad de organización; Autonomía en la toma de decisiones; Confianza en capacidades individuales y colectivas; Convicción en la viabilidad del Modelo y Comprensión en el MSF, lo cual es coincidente con algunos aspectos reportados en la literatura como elementos centrales en la Implementación del MSF: Motivación del equipo, Grado de compromiso, Madurez del Equipo, Or- ganización del equipo, Comprensión del MSF y Presencia de apoyos externos ${ }^{2}$.

Con la apropiación del MSF, por parte del Equipo del Hospital de El Carmen, éstos señalan la importancia de ver a las personas, sus familias y contexto más allá de su enfermedad “...ha sido no ver a la gente como enfermo, sino como persona, ese ba sido como el cambio porque antes venía el enfermo de algo, en cambio abora viene la señora Juanita..." lo cual es concordante con lo manifestado por Hernández ${ }^{8}$, el cual señala la implicancias de la aplicabilidad del MSF, aludiendo a una relación donde las personas son el centro de los procesos sanitarios, y los equipos de salud deben establecer una relación de respeto, empatía y compromiso con éstas.

Desde el Equipo de Salud del Hospital de El Carmen, se señalan vivencias de temor, incertidumbre, rechazo e imposición del MSF desde Servicio de Salud Provincial. Lo cual es concordante por lo reportado por estudio en torno a la vivencia de la Implementación del MSF en Equipos de APS. "La implementación del MSF en los Equipos de APS, generó vivencias de incertidumbre, desconfianza, te-

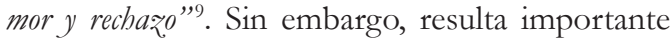
señalar que en este equipo sanitario aún cuando existió una vivencia de rechazo inicial en la implementación del MSF, también lo catalogaron como un desafío, teniendo la convicción como equipo, en la viabilidad del MSF en su Hospital "un desafío a vencernos, a no dejarnos morir ni aplastar". Este aspecto no concuerda con lo manifestado por Escobar y Vergara ${ }^{9}$, los cuales señalan que en los equipos de salud se evidenció una resistencia al cambio en la Implementación del MSF.

Finalmente, en base al actual MSF chileno (Figura 2), el cual enfatiza la importancia del contexto familiar y social en los procesos de salud y la relación que estos sistemas establecen con el equipo de atención sanitaria, las investigadoras proponen ampliar el esquema propuesto, integrando al actual modelo sanitario el concepto de "sistema de atención". Si se parte de la base de la existencia de un sistema de atención donde interactúa tanto la familia, como el equipo de atención sanitaria, (donde el MSF ha enfatizado poner a la familia como centro de los procesos de salud), el desafío actual estaría en que los propios equipos de atención sanitaria, pudieran verse como un elemento más dentro de este sistema de atención, y con ello poder estar más atentos a sus propias necesidades 


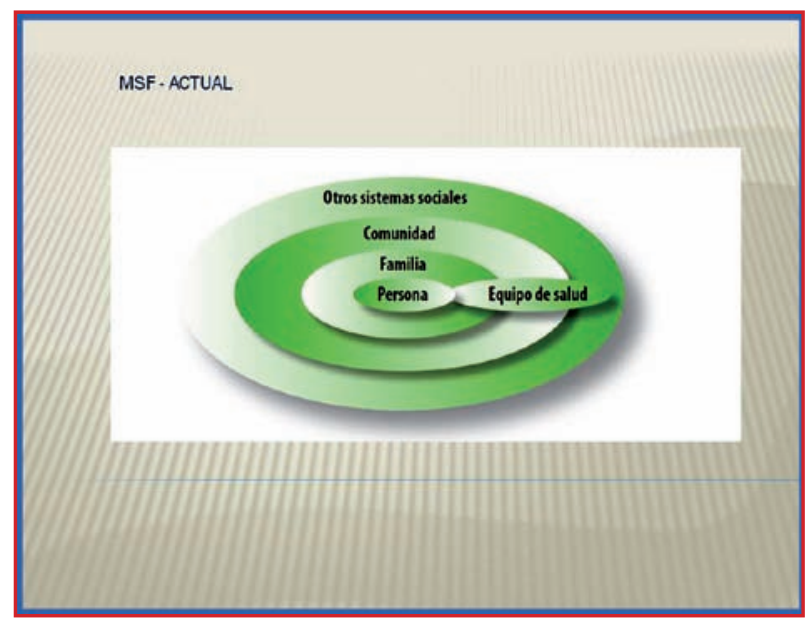

Figura 2. Modelo Salud Familiar. Fuente: MINSAL 2008.

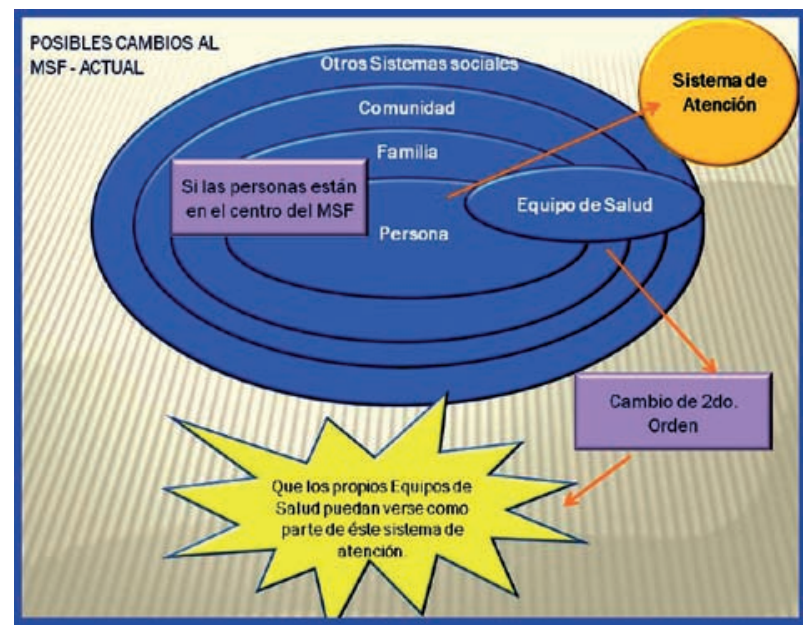

Figura 3. Modelo Salud Familiar: Sistema de Atención. Fuente: Elaboración propia.

(más allá de su rol de ayuda), estableciéndose de esta manera desde la lógica sistémica, una mirada de segundo orden, en función de los sistemas de relaciones que se establecen en los procesos de salud con el desarrollo del MSF chileno (Figura 3).

\section{LIMITACIONES}

Sólo se entrevistaron a 17 miembros del equipo de salud del Hospital de El Carmen, pudiéndose haber ampliado la muestra y con ello haber obtenido una mayor riqueza de la experiencia de este equipo de atención hospitalaria.

\section{FUTURAS LINEAS DE INVESTIGACIÓN}

Se plantea la posibilidad de realizar otros estudios en equipos de atención hospitalaria en torno a su experiencia en la implementación del MSF. A su vez, tal como las investigadoras han planteado, en el establecimiento de este "sistema" de atención, sería interesante realizar estudios en la implementación del MSF en familias atendidas por equipos con formación en el MSF, y con ello poder obtener una comprensión más integral y sistémica de los procesos de salud que en esos espacios de interrelación que se producen. 


\section{REFERENCIAS}

1. Suárez-Bustamante, M. Los Modelos de Reforma de Salud Familiar en América Latina. Journal Medical Family, 2010; 4: 61-69.

2. Depaux R, Campodonico L, Ringeling I, Segovia I. En el camino a Centro de Salud Familiar. Secretaría de Redes Asistenciales, 2008.

3. Schonhaut, L. Los desafíos de la enseñanza de pediatría ambulatoria en el contexto del Chile actual. Revista Chilena de Pediatría. 2006.77; 405-411. Santiago. doi:10.4067/S037041062006000400012.

4. Cisternas C. Experiencias de Aplicación de Redes de Salud en Chile. Centro de Gestión de Instituciones de Salud. Universidad de Talca, 2010.

5. Ministerio de Salud, 2010. Orientaciones para la
Planificación y Programación en Red año 2011. Cuadernos de redes.

6. Ministerio de Salud, 2008. Marco referencial Hospitales de la Familia y Comunidad. Subsecretaría de Redes Asistenciales. Cuaderno de Redes No 20.

7. López A, Rastello A y Rogers. "Chilenización" del Modelo de Salud Familiar en los Cesfam de Valparaíso al mes de Junio, (2002).

8. Hernández M. El enfoque sociopolítico para el análisis de las reformas sanitarias en América Latina. Revista de la Facultad Nacional de Salud Pública. Año número 19, 2001. Pp.57-70.

9. Escobar P, Vergara G. Tendencias en Salud Pública: Salud Familiar y Comunitaria y Promoción. Programa de Diplomado en Salud Pública y Salud Familiar, Universidad Austral de Chile, 2006. 